\title{
Small dark energy and stable vacuum from Dilaton-Gauss-Bonnet coupling in TMT
}

\author{
Eduardo I. Guendelman ${ }^{1, \mathrm{a}}$, Hitoshi Nishino ${ }^{2, \mathrm{~b}}$, Subhash Rajpoot ${ }^{2, \mathrm{c}}$ \\ ${ }^{1}$ Department of Physics, Ben-Gurion University of the Negev, Beer-Sheva, Israel \\ ${ }^{2}$ California State University at Long Beach, Long Beach, CA, USA
}

Received: 2 January 2017 / Accepted: 27 March 2017 / Published online: 13 April 2017

(C) The Author(s) 2017. This article is an open access publication

\begin{abstract}
In two measures theories (TMT), in addition to the Riemannian measure of integration, being the square root of the determinant of the metric, we introduce a metricindependent density $\Phi$ in four dimensions defined in terms of scalars $\varphi_{a}$ by $\Phi=\varepsilon^{\mu \nu \rho \sigma} \varepsilon_{a b c d}\left(\partial_{\mu} \varphi_{a}\right)\left(\partial_{\nu} \varphi_{b}\right)\left(\partial_{\rho} \varphi_{c}\right)\left(\partial_{\sigma} \varphi_{d}\right)$. With the help of a dilaton field $\phi$ we construct theories that are globally scale invariant. In particular, by introducing couplings of the dilaton $\phi$ to the Gauss-Bonnet (GB) topological density $\sqrt{-g} \phi\left(R_{\mu \nu \rho \sigma}^{2}-4 R_{\mu \nu}^{2}+R^{2}\right)$ we obtain a theory that is scale invariant up to a total divergence. Integration of the $\varphi_{a}$ field equation leads to an integration constant that breaks the global scale symmetry. We discuss the stabilizing effects of the coupling of the dilaton to the GB-topological density on the vacua with a very small cosmological constant and the resolution of the 'TMT Vacuum-Manifold Problem' which exists in the zero cosmological-constant vacuum limit. This problem generically arises from an effective potential that is a perfect square, and it gives rise to a vacuum manifold instead of a unique vacuum solution in the presence of many different scalars, like the dilaton, the Higgs, etc. In the non-zero cosmological-constant case this problem disappears. Furthermore, the GB coupling to the dilaton eliminates flat directions in the effective potential, and it totally lifts the vacuum-manifold degeneracy.
\end{abstract}

\section{Introduction}

There are two basic formulations of the CosmologicalConstant Problem (CCP), the first one is referred to as the 'Old Cosmological-Constant Problem' [1,2], where it was believed that the vacuum energy density of the universe was exactly zero and physicists invented mechanisms to obtain

\footnotetext{
a e-mail: guendel@bgu.ac.il

b e-mail: h.nishino@csulb.edu

c e-mail: Subhash.Rajpoot@csulb.edu
}

exactly zero vacuum energy density for the universe today. Since more recently, a different type of CCP is being considered due to the evidence for the accelerating expansion of the universe [3]. We therefore have a 'New CosmologicalConstant Problem' [4]. The problem is now not to explain the zero, but to explain a very small vacuum energy density.

The evidence of a very small vacuum energy density of the universe means that getting a zero vacuum energy density for the present universe is definitely not the full solution of the problem, although it may be a first step toward its solution. Many possibilities appear. It could be that the true vacuum of the theory still has zero vacuum energy density, but we have not reached this point yet, and that is why we see a small vacuum energy density now. Alternatively, it could be that the true vacuum state of the theory has a non-zero vacuum energy density. In this case, there could as well be many possibilities. It could be that, although there is a basic mechanism to drive the vacuum energy density to zero, some 'residual' interaction exists that is responsible for slightly shifting the vacuum energy density toward a small but non-zero value. Together with identifying a certain mechanism that drives the vacuum energy density to zero, we then consider a 'residual' interaction that provides a small vacuum energy density. Another possibility is the existence of 'cosmological see-saw mechanism', where the vacuum energy density arises as the ratio of a certain coupling constant squared divided by another coupling constant. In this scenario, if the first coupling is of the order of electroweak scale to the fourth power and the second is of the order of Planck-scale to the fourth power, the presently observed vacuum energy of the universe is obtained as a 'see-saw' effect, i.e., at $\mathcal{O}\left(M_{\mathrm{W}}^{8} / M_{\mathrm{Pl}}^{4}\right)$.

Interestingly enough, all the basic mechanisms described above can be studied in the context of the TMT. It is a mechanism able to drive the vacuum energy density of the universe to zero [5], and can also be used in a spontaneously broken scale-invariant model to obtain the 'cosmological see-saw mechanism' [6]. Still in the TMT, by introducing couplings of 
the dilaton to a topological density constructed out of gauge fields, a small 'residual' interaction can provide a small vacuum energy density (which is activated through instantons) as explored in [7].

Concerning the basic mechanism to drive the vacuum energy density to zero, we have studied a new class of theories [5], based on the idea that the action-integral may contain the new metric-independent measure of integration. For example, in four dimensions the new measure can be built from a three index field [8] as

$\Phi=\varepsilon^{\mu \nu \rho \sigma} \partial_{\mu} A_{\nu \rho \sigma}$,

or out of four scalar fields $\varphi_{a}(a=1,2,3,4)$ as

$$
\Phi=\varepsilon^{\mu \nu \rho \sigma} \varepsilon_{a b c d}\left(\partial_{\mu} \varphi_{a}\right)\left(\partial_{\nu} \varphi_{b}\right)\left(\partial_{\rho} \varphi_{c}\right)\left(\partial_{\sigma} \varphi_{d}\right)
$$

To provide parity conservation, one can choose one of the $\varphi_{a}$ to be a pseudo-scalar.

These two representations give the same results. There is another inequivalent choice for constructing an alternative measure using four Lorentz vectors and preserving local Lorentz invariance [9]. ${ }^{1}$

The $\Phi$ is a scalar density under general coordinate transformations and the action can be chosen in the form $S=$ $\int L \Phi \mathrm{d}^{4} x$. This has been applied to three different situations.

(1) Investigation of 4D gravity and matter fields models containing the new measure of integration that appears to be promising for resolving the dark-energy and dark-matter problems, the fifth-force problem, etc.

(2) Studying new type of string and brane models based on the use of a modified world-sheet/world-volume integration measure [10-14]. It allows new types of objects and effects like a spontaneously induced string tension; a classical mechanism for a charge confinement; and a Weyl-conformally invariant light-like (WILL) brane [13], obtaining promising results for black hole physics.

(3) Studying higher-dimensional realization of the idea of the modified measure in the context of the Kaluza-Klein [13] and brane [15] scenarios with the aim to solve the CCP. Finally a mechanism for supersymmetry breaking has also been found using a modified measure formulation of supergravity [16].

We start with the action

$S=\int L_{1} \Phi \mathrm{d}^{4} x+\int L_{2} \sqrt{-g} \mathrm{~d}^{4} x$,

including two Lagrangians $L_{1}$ and $L_{2}$ and two volume elements: $\Phi \mathrm{d}^{4} x$ and $\sqrt{-g} \mathrm{~d}^{4} x$, respectively. For constructing the field theory with the action (1.3), we make only the basic additional assumption that $L_{1}$ and $L_{2}$ are independent of the measure fields $\varphi_{a}$. The action (1.3) is invariant under volume

\footnotetext{
1 This third approach opens new possibilities, not fully explored in detail as yet.
}

preserving diffeomorphisms. Besides, it is invariant (up to an integral of a total divergence) under the infinite-dimensional group of shifts of the measure fields $\varphi_{a}: \varphi_{a} \rightarrow \varphi_{a}+f_{a}\left(L_{1}\right)$, where $f_{a}\left(L_{1}\right)$ are arbitrary differentiable functions of the Lagrangian $L_{1}$. We can proceed in the first-order formalism where all fields, including the metric $g_{\mu \nu}$ (or vierbeins $e_{a \mu}$ ), connection coefficients (or spin-connection $\omega_{\mu}^{a b}$ ) and the measure fields $\varphi_{a}$ are treated as independent dynamical variables. All the relations between them follow from equations of motion. The field theory based on the above-listed assumptions, we call TMT.

It turns out that the measure fields $\varphi_{a}$ affect the theory only via the ratio of the two measures

$$
\zeta \equiv \frac{\Phi}{\sqrt{-g}}=e^{-1} \Phi,
$$

where $e \equiv \operatorname{det}\left(e_{\mu}{ }^{m}\right)=\sqrt{-g}$, and $\zeta$ is a scalar field with zero scaling-weight that is determined by a constraint in the form of an algebraic equation which is a consistency condition of the equations of motion.

TMT models naturally avoid the fifth-force problem [17] since the coupling of the dilaton to 'normal matter', which is more dense than the vacuum energy density, is negligibly small and naturally provides a ground state with zero vacuum energy [4], since the effective potential is generically a perfect square. So when the function to be squared is zero and we take a linear correction about this point, the resulting effective potential, close to the point where it vanishes, will be proportional to the square of the deviation of the field from the point where the effective potential vanishes. Therefore, the point where the resulting effective potential vanishes is generically also a minimum. One should also notice that a structure similar to the TMT has been found in the Hodgedual formulation of supergravity theories [18]. TMTs also have many points of similarity with the 'Lagrange Multiplier Gravity (LMG)' [19,20]. The Lagrange-multiplier field in LMG enforces the condition that a certain function be zero. In the TMT this is equivalent to the constraint that requires some Lagrangian to be constant. The two measure models presented here, are different from the LMG models of $[19,20]$, and provide us with an arbitrary constant of integration for the value of a given Lagrangian, this constant of integration, if non-zero, can generate spontaneous symmetry breaking of scale invariance, which is present in the theory for example. To achieve a similar result in the Lagrangemultiplier method, we would find that it cannot respect the full scale symmetry of the theory, this symmetry would have to be broken at the level of the action, but the TMT method does not have the scale symmetry of the theory respected by the action and it can be broken only after integration of the equations of motion.

The introduction of constraints can cause Dirac fields to contribute to dark energy [17] or scalar fields to behave like 
dust in [13] and thus a dust behavior accompanied by a floating dark-energy component can be obtained in TMT [21-24].

TMTs can also be used to construct non-singular 'emergent' scenarios [25-27] for the early universe that existed since arbitrarily large early times in the form of a stable Einstein universe. This phase then gets transformed into an inflationary phase and subsequently into a slowly accelerating one. The requirement that the early phase might exist can impose restrictions on the possible values of the cosmological constant at the end [28].

The structure of TMT has been generalized in the dynamical space time theory $[29,30]$, where instead of demanding that a certain Lagrangian be constant, we require that a certain energy momentum be covariantly conserved. In the case this energy momentum is proportional to the metric, we recover the TMT in the second-order formulation. To implement this covariant conservation of a certain energy momentum tensor, we must introduce a four vector. When this four vector in restricted to be a gradient, we get a non conservation of the energy momentum tensor, which gives rise a conserved total energy momentum of the system but not to the separate conservation of DE and DM energy momentum tensors, which now interact in a diffusive fashion [31].

Recently a lot of interest has been attracted by the socalled mimetic dark matter model proposed in $[32,33]$. The latter employs a special covariant isolation of the conformal degree of freedom in Einstein gravity, whose dynamics mimics cold dark matter as a pressure-less dust. Important questions concerning the stability of mimetic gravity are studied in Refs. [34,35] also a formulates a generalized mimetic tensor-vector-scalar mimetic gravity which avoids those problems is studied. In [36] the idea is applied to inflationary scenarios.

Most versions of the mimetic gravity, except for [34] appears equivalent to a special kind of Lagrange-multiplier theory or TMT models that were known before, where the simple constraint that the kinetic term of a scalar field be constant. This of course gives identical results to a very special TMT, where the Lagrangian that couples to the new measure is the kinetic term of this scalar field.

In this paper we will show how global scale invariance can be maintained by a linear coupling of the dilaton to the Gauss-Bonnet (GB) scalar density and how this can provide stabilization of the vacuum in TMT for the case of a small cosmological constant. Due to the scale invariance, the GB coupling of the dilaton is restricted to a linear form. This situation of non-zero vacuum energy does not have the problem faced in the case of the zero cosmological constant, where there is a huge degeneracy of the vacuum, and the vacuum state is a manifold rather than a unique state. Furthermore the linear coupling of the dilaton to the GB-scalar further eliminates flat directions even in the multi-field case containing fields, such as a dilaton, the Higgs fields, etc.
Some authors [37] have used a scalar field coupled to a GB-density in cosmology. However, their methodology is quite different from ours. For example, in our case scale invariance restricts the coupling to the GB-density to be only linear in the dilaton field, while the authors in [37] feel free to choose any function that will eventually provide the desired solutions. In fact, the full-structure of the theory is rigorously derived, with no room for 'string-inspired' argument. Additionally, we focus for the moment only on the vacuum state of the universe, while the subject of [37] is a late-time universe transition from a scaling-matter era to a dark-energy universe. Those issues concerning the full history of the universe in our model will be the subject of future studies.

This paper is organized as follows: in the next section, we talk about the stability of scale-invariant TMT without any dilaton-GB coupling. In Sect. 3, we introduce a dilaton-GB coupling and study how the GB coupling eliminates vacuum flat directions. Discussion and conclusion will be given in Sect. 4.

\section{Stability issues in scale-invariant TMT without dilaton couplings to GB-scalar}

We will study first the dynamics of a scalar field $\phi$ interacting with gravity, without considering couplings to a GB-density. We start with the following action:

$$
\begin{aligned}
& S=\int L_{2} \sqrt{-g} \mathrm{~d}^{4} x+\int L_{1} \Phi \mathrm{d}^{4} x, \\
& L_{2} \equiv U(\phi), \quad L_{1} \equiv-\frac{1}{\kappa} R(\Gamma, g)+\frac{1}{2} g^{\mu \nu}\left(\partial_{\mu} \phi\right)\left(\partial_{\nu} \phi\right)-V(\phi), \\
& R(\Gamma, g) \equiv g^{\mu \nu} R_{\mu \nu}(\Gamma), \quad R_{\mu \nu}(\Gamma) \equiv R_{\mu \rho \nu}{ }^{\rho}(\Gamma), \\
& R_{\mu \nu \rho}{ }^{\sigma}(\Gamma) \equiv+\partial_{\mu} \Gamma_{\nu \rho}{ }^{\sigma}-\partial_{\nu} \Gamma_{\mu \rho}{ }^{\sigma}-\Gamma_{\mu \rho}{ }^{\tau} \Gamma_{\nu \tau}{ }^{\sigma}+\Gamma_{\nu \rho}{ }^{\tau} \Gamma_{\mu \tau}{ }^{\sigma} .
\end{aligned}
$$

In the variational principle, $\Gamma_{\mu \nu}{ }^{\rho}, g_{\mu \nu}$, the measure-field scalars $\varphi_{a}$ and the 'matter'-scalar field $\phi$ are all to be treated as independent variables. It results in equations that allow us to solve some of these variables in terms of others. Treating the connection independent of the metric is what is referred to as 'first-order formalism'. If the connection is given by the Christoffel symbol, this is referred as the 'second-order formulation'. It should be pointed out that the characterization of these two procedures as merely two different formalisms, leading to the same result is not correct. Indeed, except for the special (although very important) case of general relativity, these two procedures originate inequivalent theories, and care must be exercised if equivalence is sought.

We can implement global scale invariance in this model for very special exponential form for the $U(\phi)$ and $V(\phi)$ potentials. Indeed, if we perform the global scale transformation $g_{\mu \nu} \rightarrow e^{\theta} g_{\mu \nu}(\theta=$ const. $)$, then there is invariance 
provided $V(\phi)$ and $U(\phi)$ are of the form

$$
V(\phi)=f_{1} e^{\alpha \phi}, \quad U(\phi)=f_{2} e^{2 \alpha \phi},
$$

and $\varphi_{a}$ is transformed according to $\varphi_{a} \rightarrow \lambda_{a b} \varphi_{b}$, which implies that $\Phi \rightarrow \operatorname{det}\left(\lambda_{a b}\right) \Phi \equiv \lambda \Phi$, such that $\lambda=e^{\theta}$ and $\phi \rightarrow \phi-\theta / \alpha$.

Let us begin by considering the equations which are obtained from the variation of the fields that appear in the measure, i.e. the $\varphi_{a}$-fields. We obtain $A_{a}^{\mu} \partial_{\mu} L_{1}=0$, where $A_{a}^{\mu}=\varepsilon^{\mu \nu \rho \sigma_{\varepsilon}} \varepsilon_{a b c d}\left(\partial_{\nu} \varphi_{b}\right)\left(\partial_{\rho} \varphi_{c}\right)\left(\partial_{\sigma} \varphi_{d}\right)$, since $\operatorname{det}\left(A_{a}^{\mu}\right)=$ $\left(4 ! / 4^{4}\right) \Phi^{3}$. If $\Phi \neq 0$, we obtain $\partial_{\mu} L_{1}=0$, or

$$
L_{1}=-\frac{1}{\kappa} R(\Gamma, g)+\frac{1}{2} g^{\mu \nu}\left(\partial_{\mu} \phi\right)\left(\partial_{\nu} \phi\right)-V \doteq M,
$$

where $M$ is a constant, and the symbol $\doteq$ stands for a field equation. Notice that this equation breaks spontaneously the global scale invariance of the theory, since the left hand side has a non-trivial transformation under scale transformations, while the right hand side is equal to $M$, a constant that after we integrate the equations, is fixed and cannot be changed. Therefore, for any $M \neq 0$ we have obtained spontaneous breaking of scale invariance.

By the field equations of $g^{\mu \nu}, \phi$ and $\Gamma_{\mu \nu}{ }^{\rho}$ out of the total action $S$ we get

$\Phi\left[-\frac{1}{\kappa} R_{\mu \nu}(\Gamma)+\frac{1}{2}\left(\partial_{\mu} \phi\right)\left(\partial_{\nu} \phi\right)\right]-\frac{1}{2} \sqrt{-g} U(\phi) g_{\mu \nu} \doteq 0$,

$\partial_{\mu}\left(\Phi g^{\mu \nu} \partial_{\nu} \phi\right)-\sqrt{-g} U^{\prime}(\phi)+\Phi V^{\prime}(\phi) \doteq 0$,

$$
\begin{aligned}
& \Gamma_{\rho}{ }^{v \mu}+\Gamma^{\mu} \rho^{v}-g^{\mu v} \Gamma_{\rho \sigma}{ }^{\sigma}-\delta_{\rho}^{\mu} \Gamma_{\sigma}{ }^{\sigma v}+\partial_{\rho} g^{\mu v} \\
& -\delta_{\rho}^{\mu} \partial_{\sigma} g^{\sigma v}+g^{\mu v} \partial_{\rho}(\ln \Phi)-\delta_{\rho}^{\mu} g^{\nu \sigma} \partial_{\sigma}(\ln \Phi) \\
& +T_{\rho}{ }^{\mu v}+\delta_{\rho}{ }^{\mu} T^{v}{ }_{\sigma}{ }^{\sigma}-T^{\rho v \mu} \doteq 0,
\end{aligned}
$$

where $T_{\mu \nu}{ }^{\rho} \equiv \Gamma_{\mu \nu}{ }^{\rho}-\Gamma_{\nu \mu}{ }^{\rho}$ is the torsion tensor. In our present formulation, we look only for torsion-free solutions, so that the last three $T$-linear terms in $(2.4 \mathrm{c})$ are ignored, and we restrict $\Gamma_{\mu \nu}{ }^{\rho}=\Gamma_{(\mu \nu)}{ }^{\rho}$.

Solving (2.4a) for $R(\Gamma)=g^{\mu \nu} R_{\mu \nu}(\Gamma)$, and substituting it into (2.3), we obtain a constraint that allows us to solve for the ratio $\zeta$ of the two measures:

$\zeta \equiv \frac{\Phi}{\sqrt{-g}} \doteq \frac{2 U(\phi)}{M+V(\phi)}$.

As for the $\Gamma$-field equation, we substitute

$\Gamma_{\mu \nu}{ }^{\rho}=\left\{\begin{array}{l}\rho \\ \mu \nu\end{array}\right\}+\Sigma_{\mu \nu}{ }^{\rho} \quad\left(\Sigma_{\mu \nu}{ }^{\rho}=\Sigma_{\nu \mu}{ }^{\rho}\right)$,

into $(2.4 \mathrm{c})$ to get

$$
\begin{aligned}
& \Sigma_{\mu \nu \rho}+\Sigma_{\rho \mu \nu}-g_{\nu \rho} \Sigma_{\mu \sigma} \sigma+2 g_{\mu \rho} \Sigma_{\nu \sigma}{ }^{\sigma}+g_{\nu \rho} \partial_{\mu}(\ln \zeta) \\
& -4 g_{\mu \rho} \partial_{\nu}(\ln \zeta) \doteq 0 .
\end{aligned}
$$

This is solved for $\Sigma$ as

$$
\Sigma_{\mu \nu \rho} \doteq+\frac{1}{2} \zeta^{-1}\left(g_{\mu \rho} \partial_{\nu} \zeta+g_{\nu \rho} \partial_{\mu} \zeta-g_{\mu \nu} \partial_{\rho} \zeta\right)
$$

To get the physical content of the theory, it is best to consider variables that have a well-defined dynamical interpretation. The original metric does not have a non-zero canonical momenta in the first-order formalism as no derivatives of the metric appear in the Lagrangian. All derivatives appear in the connections, which are the fundamental dynamical variables of the theory. The canonical momenta of these connections are functions of $\bar{g}_{\mu \nu}$, where

$\bar{g}_{\mu \nu}=\zeta g_{\mu \nu}$,

where $\zeta$ is defined by (2.5). Interestingly enough, working with $\bar{g}_{\mu \nu}$ is the same as going to the 'Einstein conformal frame'. It turns out that in terms of $\bar{g}_{\mu \nu}$ the non-Riemannian contribution $\Sigma_{\mu \nu}{ }^{\rho}$ disappears from the equations, that is, $\bar{\Gamma}_{\mu \nu}{ }^{\rho}=\left.\left\{\begin{array}{l}\rho \\ \mu \nu\end{array}\right\}\right|_{\bar{g}_{\sigma \tau}}$. This is because the connection can be recast as the Christoffel symbol of the metric $\bar{g}_{\mu \nu} \cdot{ }^{2}$ In terms of $\bar{g}_{\mu \nu}$ the equations of motion for the metric can then be written in the Einstein form,

$\bar{R}_{\mu \nu}(\bar{g})-\frac{1}{2} \bar{g}_{\mu \nu} \bar{R}(\bar{g}) \doteq \frac{\kappa}{2} T_{\mu \nu}^{\text {eff }}(\phi)$,
$T_{\mu \nu}^{\text {eff }}(\phi) \doteq\left(\partial_{\mu} \phi\right)\left(\partial_{\nu} \phi\right)-\frac{1}{2} \bar{g}_{\mu \nu} \bar{g}^{\rho \sigma}\left(\partial_{\rho} \phi\right)\left(\partial_{\sigma} \phi\right)+\bar{g}_{\mu \nu} V_{\text {eff }}(\phi)$,

$V_{\mathrm{eff}}(\phi) \doteq \frac{1}{4 U(\phi)}(V+M)^{2}$,

$\Gamma_{\mu \nu}{ }^{\rho} \doteq \bar{\Gamma}_{\mu \nu}{ }^{\rho}(\bar{g})=\left.\left\{\begin{array}{l}\rho \\ \mu \nu\end{array}\right\}\right|_{\bar{g}_{\sigma \tau}}$,

where we define $\bar{R}_{\mu \nu}(\bar{g}) \equiv R_{\mu \nu}(\bar{\Gamma}(\bar{g})), \bar{R}(\bar{g}) \equiv R(\bar{\Gamma}(\bar{g})) \equiv$ $\bar{g}^{\mu \nu} \bar{R}_{\mu \nu}(\bar{g})$.

Using the metric $\bar{g}^{\alpha \beta}$, the $\phi$-field Eq. (2.4b) becomes

$$
\frac{1}{\sqrt{-\bar{g}}} \partial_{\mu}\left(\bar{g}^{\mu \nu} \sqrt{-\bar{g}} \partial_{\nu} \phi\right)+V_{\text {eff }}^{\prime}(\phi) \doteq 0 .
$$

In this case the vacuum is obtained for $V+M=0$, where $V_{\text {eff }}=0$ and $V_{\text {eff }}^{\prime}=0$ also, provided $V^{\prime}$ is finite and $U \neq 0$ there. This means that the vacuum with zero cosmologicalconstant state is achieved without any fine-tuning. That is, independent of whether we add to $V$ a constant piece, or whether we change the value of $M$, as long as there is still a point where $V+M=0$, then still $V_{\text {eff }}=0$ and $V_{\text {eff }}^{\prime}=0$. This is the basic feature that characterizes the TMT and allows it to solve the 'old' CCP.

If $V(\phi)=f_{1} e^{\alpha \phi}$ and $U(\phi)=f_{2} e^{2 \alpha \phi}$ as required by global scale invariance, we obtain from (2.10c)

$$
V_{\mathrm{eff}}=\frac{1}{4 f_{2}}\left(f_{1}+M e^{-\alpha \phi}\right)^{2} \text {. }
$$

2 This is also understandable from the viewpoint of Weyl rescaling: the factor $\Phi \equiv \zeta \sqrt{-g}$ in front of the scalar curvature can be completely absorbed into the Weyl rescaling of the metric. 


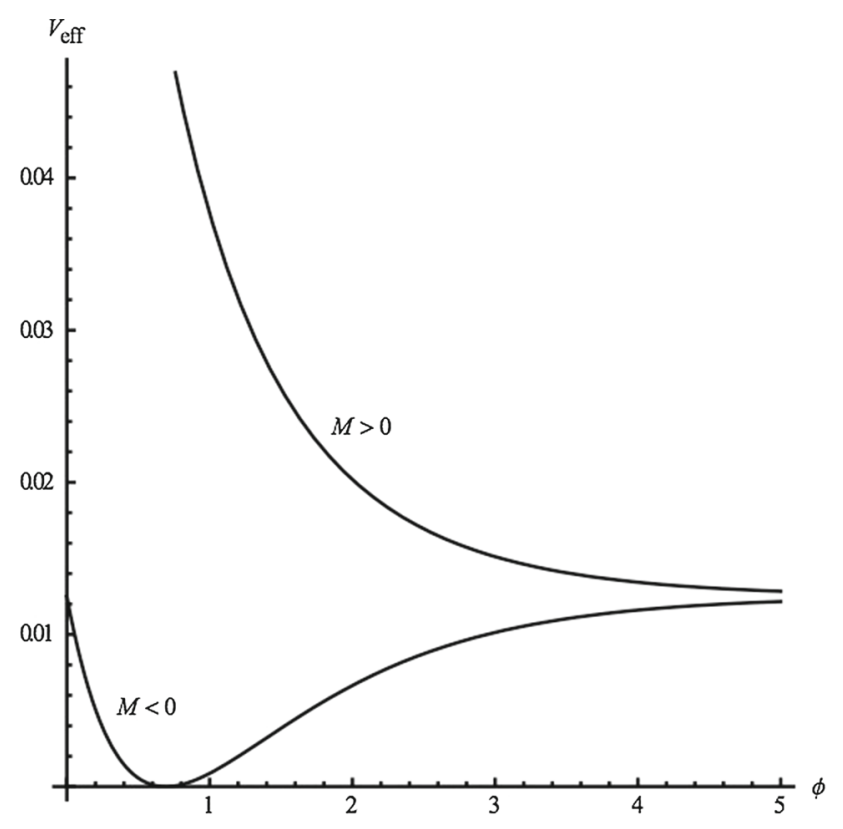

Fig. 1 Graph of $V_{\text {eff }}$ for $M>0$ and $M<0$. The minimum appears for the graph $M<0$

Since we can always perform the transformation $\phi \rightarrow-\phi$ we can choose by convention $\alpha>0$. We then see that as $\phi \rightarrow \infty, V_{\text {eff }} \rightarrow f_{1}^{2} /\left(4 f_{2}\right)=$ const., providing an infinite flat region for the effective potential (see Fig. 1 below). Also a minimum is achieved at zero cosmological constant for the case $f_{1} / M<0$ at the point

$$
\phi_{\min }=-\frac{1}{\alpha} \ln \left|\frac{f_{1}}{M}\right| \text {. }
$$

For the case when $f_{1} / M>0$, the effective potential never reaches zero as in Fig. 1.

In both cases the asymptotic value of $V_{\text {eff }}$ as $\phi \rightarrow \infty$ is, according to Eq. (2.12), $V_{\text {eff }}(\infty)=f_{1}^{2} /\left(4 f_{2}\right)$, which can be made very small by means of a see-saw mechanism. By considering $f_{1} \approx \mathcal{O}\left(M_{\mathrm{EW}}^{4}\right)$ and $f_{2} \approx \mathcal{O}\left(M_{\text {Planck }}^{4}\right)$, the observed vacuum energy is indeed of $\mathcal{O}\left(M_{\mathrm{EW}}^{8} / M_{\text {Planck }}^{4}\right)$. So this flat region is indeed a good candidate for representing the vacuum of the present universe if this see-saw mechanism is invoked. There is the issue, however, that there is no particular point at which the dilaton is stabilized, leading to the appearance of a massless field. Notice that in spite of the existence of a massless field, TMT models naturally avoid the fifth-force problem [38], since the coupling of the dilaton to 'normal matter', which is more dense than the vacuum energy density, is negligibly small, but still the vacuum is still undetermined, since all values in the infinite flat region of the potential (2.12) appear equivalent

If we have several scalars, however, like a dilaton and a Higgs field, the sum of the potentials of each of the fields that couple to the measure $\Phi$ squared will appear in the effective potential and the vacuum with zero cosmological constant will be realized by a manifold of infinite possibilities, where the sum of the potentials of each of the fields vanishes. So it is of interest to see how this degeneracy can be lifted.

As will be demonstrated in the next section, enriching the scale-invariant TMT with a linear coupling of the dilaton with the GB-density, does indeed lift both of the degeneracies described above. In the case of a single dilaton field, this coupling can determine where in the flat region of the effective potential the dilaton must reside in the vacuum state, and in the case of the vacuum with zero cosmological constant, the manifold degeneracy will be lifted because in addition to the condition that the sum of the potentials of each of the fields that couple to the measure $\Phi$ be zero, another equation from the dilaton-field equation will appear that will assist in the process. All the details are explicitly presented in what follows.

\section{Introducing the dilaton-GB coupling and stabilizing the vacuum-degeneracy problem}

Coupling the dilaton to the GB-density, which is a total derivative, provide us with an interesting stabilization property in several situations in TMT in the cases of zero or small cosmological constant.

In this paper we consider a linear coupling of the dilaton to the GB-scalar:

$$
G \equiv R_{\mu \nu \rho \sigma}(\Gamma) R^{\mu \nu \rho \sigma}(\Gamma)-4 R_{\mu \nu}(\Gamma) R^{\mu \nu}(\Gamma)+R^{2}(\Gamma),
$$

Only for the GB-term, we restrict later the affinity $\Gamma$ to the Christoffel connection $\left\{\begin{array}{c}\mu \\ v \rho\end{array}\right\}$. For the other parts of the Lagrangian we leave the connection to be defined by the equations of motion of the connection. In other words, we adopt the first-order formulation for the Lagrangians $L_{1}$ and $L_{2}$, while we have the second-order formulation for our GBterm. ${ }^{3}$

There are good reasons why the GB-density is considered in the second-order formulation. Because when we consider a torsion [40], or other non-Riemannian structures in the firstorder formulation, the GB-density becomes non-topological. Since the invariance of the action under scale transformations requires the GB-term to be topological, we must consider only the GB-term in the second-order formalism.

In our work, the GB-term in the second-order formalism is coupled linearly to the dilaton and the rest of the action formulated in the first-order formalism. They give a rise to second-order differential equations, and the ratio between the new measure and the Riemannian measure is determined by

\footnotetext{
${ }_{3}$ In passing, we note that terms of the type $\sqrt{-g} G \ln G$ were considered in [39].
} 
an algebraic constraint. This way, the new measure does not introduce new degrees of freedom. In this case, we achieve invariance under global scale-invariance transformations up to a total derivative. This total derivative can give a nontrivial contribution in the presence of gravitational instantons $[41,42]$.

We will now study the dynamics of a scalar field $\phi$ interacting with gravity as given by the following action:

$S=\int L_{2} \sqrt{-g} \mathrm{~d}^{4} x+\int L_{1} \Phi \mathrm{d}^{4} x+\int N \phi \sqrt{-g} G \mathrm{~d}^{4} x$,

$L_{2} \equiv U(\phi), \quad L_{1} \equiv-\frac{1}{\kappa} R(\Gamma, g)+\frac{1}{2} g^{\mu \nu}\left(\partial_{\mu} \phi\right)\left(\partial_{\nu} \phi\right)-V(\phi)$,

$R(\Gamma, g) \equiv g^{\mu \nu} R_{\mu \nu}(\Gamma), \quad R_{\mu \nu}(\Gamma) \equiv R_{\mu \rho \nu}{ }^{\rho}(\Gamma)$,

$R_{\mu \nu \rho}{ }^{\sigma}(\Gamma) \equiv+\partial_{\mu} \Gamma_{\nu \rho}{ }^{\sigma}-\partial_{\nu} \Gamma_{\mu \rho}{ }^{\sigma}-\Gamma_{\mu \rho}{ }^{\tau} \Gamma_{\nu \tau}{ }^{\sigma}+\Gamma_{\nu \rho}{ }^{\tau} \Gamma_{\mu \tau}{ }^{\sigma}$,

where $N$ is a non-zero real constant. In the variational principle, except for the GB-term, $\Gamma_{\mu \nu}{ }^{\rho}, g_{\mu \nu}$, the measure-field scalars $\varphi_{a}$, and the 'matter'-scalar field $\phi$ are all to be treated as independent variables. In the GB-term, only $g_{\mu \nu}$ and $\phi$ are involved, because $R_{\mu \nu \rho}{ }^{\sigma}(g) \equiv R_{\mu \nu \rho}{ }^{\sigma}(\{\})$. In other words, the Lagrangians $L_{1}$ and $L_{2}$ are in the first-order formulation, while the GB-term is in the second-order formulation.

For simplicity of computation, it is advantageous to use the Lorentz connection $\omega_{\mu}{ }^{r s}$, corresponding to the Christoffel connection:

$$
\begin{aligned}
& \omega_{m r s}(e) \equiv-\frac{1}{2}\left(C_{m r s}-C_{m s r}-C_{r s m}\right), \\
& C_{\mu \nu}{ }^{r} \equiv \partial_{\mu} e_{\nu}^{r}-\partial_{\nu} e_{\mu}^{r},
\end{aligned}
$$

so the Lorentz-covariant derivative acts like $D_{\mu}(\omega) X_{m} \equiv$ $\partial_{\mu} X_{m}-\omega_{\mu m}^{n}(e) X_{n}$ for an arbitrary vector $X_{m}$ with a local Lorentz index. Therefore, the Lorentz curvature is

$$
\begin{aligned}
R_{\mu \nu}{ }^{r s}(\omega(e)) \equiv & +\partial_{\mu} \omega_{\nu}^{r s}(e)-\partial_{\nu} \omega_{\mu}^{r s}(e) \\
& -\omega_{\mu}{ }^{r t}(e) \omega_{\nu t}{ }^{s}(e)+\omega_{\nu}{ }^{r t}(e) \omega_{\mu t}{ }^{s}(e) .
\end{aligned}
$$

Accordingly, there is a direct relationship between $R_{\mu \nu \rho} \sigma(\{\})$ $\equiv R_{\mu \nu \rho}{ }^{\sigma}(g)$ and $R_{\mu \nu \rho}{ }^{\sigma}(\omega(e))$ :

$$
R_{\mu \nu \rho}^{\sigma}(g)=e_{\rho r} e_{s}^{\sigma} R_{\mu \nu}^{r s}(\omega(e))
$$

which is confirmed under the 'metricity' or 'vierbeinpostulate': $D_{\mu} e_{\nu}^{r} \equiv \partial_{\mu} e_{\nu}^{r}-\omega_{\mu}^{r s} e_{\nu s}-\left\{\begin{array}{l}\sigma \\ \mu \nu\end{array}\right\} e_{\sigma}^{r} \equiv$ $D_{\mu}(\omega) e_{\nu}^{r}-\left\{\begin{array}{l}\sigma \\ \mu \nu\end{array}\right\} e_{\sigma}^{r}=0$ in the second-order formulation. Relevantly, there are two ways to express our GB-term in (3.2a) in the second-order formulation:

$$
\begin{aligned}
G & =R_{\mu \nu \rho \sigma}(g) R^{\mu \nu \rho \sigma}(g)-4 R_{\mu \nu}(g) R^{\mu \nu}(g)+R^{2}(g) \\
& =-\frac{1}{4} e^{-1} \epsilon^{\mu \nu \rho \sigma} e_{m n r s} R_{\mu \nu}^{m n}(\omega(e)) R_{\rho \sigma}{ }^{r s}(\omega(e)) .
\end{aligned}
$$

Equation (3.6b) simplifies the computation, because of there being no involvement of the metric or vierbein other than $\omega(e)$.

As alluded to before, we can have global scale invariance in this model for the very special exponential form for the $U(\phi)$ and $V(\phi)$ potentials (2.2). Indeed, if we perform the global scale transformation $g_{\mu \nu} \rightarrow e^{\theta} g_{\mu \nu} \quad(\theta=$ const. $)$, then there is invariance provided $V(\phi)$ and $U(\phi)$ are of the form (2.2). ${ }^{4}$

Let us begin by considering the equations that are obtained from the variation of the fields that appear in the measure, i.e. the $\varphi_{a}$ fields. We obtain $A_{a}{ }^{\mu} \partial_{\mu} L_{1}=0$ where $A_{a}{ }^{\mu}=\varepsilon^{\mu \nu \rho \sigma} \varepsilon_{a b c d}\left(\partial_{\nu} \varphi_{b}\right)\left(\partial_{\rho} \varphi_{c}\right)\left(\partial_{\sigma} \varphi_{d}\right)$. Now $\operatorname{det}\left(A_{a}^{\mu}\right)=$ $\left(4 ! / 4^{4}\right) \Phi^{3}$. If $\Phi \neq 0$, we obtain the same result as (2.3).

The field equations of $g^{\mu \nu}, \phi$ and $\Gamma_{\mu \nu}{ }^{\rho}$ are

$$
\begin{aligned}
& \Phi\left[-\frac{1}{\kappa} R_{\mu \nu}(\Gamma)+\frac{1}{2}\left(\partial_{\mu} \phi\right)\left(\partial_{\nu} \phi\right)\right]-\frac{1}{2} \sqrt{-g} U(\phi) g_{\mu \nu} \\
& \left.\quad+4 N e_{(} \mu^{m} e_{\nu}\right)^{n} D_{r}(\omega)\left[e^{*} R^{*}{ }_{m}^{s}{ }_{n}^{r} e_{s}^{\sigma}\left(\partial_{\sigma} \phi\right)\right] \\
& \quad+2 N g_{\mu \nu} D_{m}(\omega)\left[e G^{m \rho} \partial_{\rho} \phi\right] \doteq 0, \\
& \partial_{\mu}\left(\Phi g^{\mu \nu} \partial_{\nu} \phi\right)-\sqrt{-g} U^{\prime}(\phi)+\Phi V^{\prime}(\phi)-N \sqrt{-g} G \doteq 0,
\end{aligned}
$$

$$
\begin{aligned}
& \Gamma_{\rho}{ }^{v \mu}+\Gamma_{\rho}^{\mu} \rho^{v}-g^{\mu v} \Gamma_{\rho \sigma}{ }^{\sigma}-\delta_{\rho}^{\mu} \Gamma_{\sigma}{ }^{\sigma v}+\partial_{\rho} g^{\mu v}-\delta_{\rho}^{\mu} \partial_{\sigma} g^{\sigma v} \\
& \quad+g^{\mu v} \partial_{\rho}(\ln \Phi)-\delta_{\rho}^{\mu} g^{v \sigma} \partial_{\sigma}(\ln \Phi)+T_{\rho}{ }^{\mu v} \\
& \quad+\delta_{\rho}^{\mu} T_{\sigma}^{v}{ }^{\sigma}-T^{\rho v \mu} \doteq 0,
\end{aligned}
$$

where $G_{m n} \equiv R_{m n}-(1 / 2) \eta_{m n} R$ is the Einstein tensor, and ${ }^{*} R_{m n r s}^{*} \equiv(1 / 4) \epsilon_{m n}{ }^{t u} \epsilon_{r s}{ }^{v w} R_{t u v w}$.

Note that the last two terms in (3.7a) are from the variation of the GB-term with at least one derivative on $\phi$. Under our principle of choosing the second-order formulation for the GB-term, the GB-term has no $\Gamma$-field, so that the $\Gamma$-field equation $(3.7 \mathrm{c})$ is exactly the same as $(2.4 \mathrm{c})$. As in the last section, we can drop the $T$-linear terms if we ignore torsion.

For a slow-varying or constant $\phi$, the last two terms in (3.7a) can be ignored. Solving (3.3) for $R(\Gamma)=g^{\mu \nu} R_{\mu \nu}(\Gamma)$, and substituting it into (2.3), we obtain a constraint that allows us to solve for the ratio $\zeta$ of the two measures as

$$
\zeta \doteq \frac{2 U(\phi)}{M+V(\phi)}+\mathcal{O}\left(\partial_{\nu} \phi\right)
$$

Here the last term of $\mathcal{O}\left(\partial_{\nu} \phi\right)$ is from the last two terms in (3.7a) from the GB-term with at least one derivative on $\phi$, which we ignore, since we assume that $\phi$ is slowly varying or constant.

\footnotetext{
4 The case without coupling to the GB-topological density was studied in Ref. [43].
} 
To get the physical content of the theory, it is best to consider variables that have a well-defined dynamical interpretation. The original metric does not have a non-zero canonical momenta in the first-order formalism as no derivatives of such metric appear in the Lagrangian. All derivatives appear in the connections, which are the fundamental dynamical variables of the theory. The canonical momenta of those connections are functions of $\bar{g}_{\mu \nu}$ given by (2.9) where $\zeta$ is defined by (3.8). As in the last section, we can go to the 'Einstein Conformal Frame', and use the metric $\bar{g}_{\mu \nu}$ instead of $g_{\mu \nu}$. As in the previous case without the GB-term, all $\zeta$-dependent terms in $\Gamma_{\mu \nu}{ }^{\rho}$ are absorbed into the Weyl rescaling (2.9), and therefore the affinity is entirely expressed by the Christoffel symbol: $\left.\Gamma_{\mu \nu}{ }^{\rho} \doteq \bar{\Gamma}_{\mu \nu}{ }^{\rho} \doteq\left\{\begin{array}{c}\rho \\ \mu \nu\end{array}\right\}\right|_{\bar{g}_{\sigma \tau}}$.

Eventually, the forms of (2.10a) and (2.10c) are intact, while $(2.10 \mathrm{~b})$ is now modified to

$$
\begin{aligned}
T_{\mu \nu}^{\mathrm{eff}}(\phi)= & \left(\partial_{\mu} \phi\right)\left(\partial_{\nu} \phi\right)-\frac{1}{2} \bar{g}_{\mu \nu} \bar{g}^{\rho \sigma}\left(\partial_{\rho} \phi\right)\left(\partial_{\sigma} \phi\right) \\
& +\bar{g}_{\mu \nu} V_{\mathrm{eff}}(\phi)+\mathcal{O}\left(\partial_{\nu} \phi\right) .
\end{aligned}
$$

Using the metric $\bar{g}^{\rho \sigma}$, the $\phi$-field equation (3.7b) becomes

$$
\begin{aligned}
& \frac{1}{\sqrt{-\bar{g}}} \partial_{\mu}\left(\bar{g}^{\mu \nu} \sqrt{-\bar{g}} \partial_{\nu} \phi\right)+V_{\mathrm{eff}}^{\prime}(\phi)+N G(\bar{g}) \\
& \quad+N \mathcal{O}\left(\partial_{\nu} \phi\right) \doteq 0 .
\end{aligned}
$$

The case $N \rightarrow 0$ coincides with the result in Sect. 2. Also, notice that in the above equation everything must be calculated using the metric in the Einstein frame, the $\mathcal{O}\left(\partial_{\nu} \phi\right)$ terms originate from the fact that the GB has a simple scaling transformation for a rescaling of the metric, but for a space time dependent scaling (through the space time dependence of $\phi$ ) these terms will appear, but are irrelevant for the case of constant $\phi$. However, the non-zero GB-term changes this picture (cf. Fig. 1). Because of the additional $N$-term, the scalar field may be determined to assume a constant value at a value different from the extremum of $V_{\text {eff }}$, Furthermore, even if the effective potential $V_{\text {eff }}$ has no extremum, the equation of the $\phi$-field can be stabilized at a certain value

$$
V_{\text {eff }}^{\prime}(\langle\phi\rangle)+N G \doteq 0 .
$$

This equation follows from (3.10), since we take $\phi$ to be slowly varying or constant.

As for the GB-contribution, two remarks are in order: First, the $\Gamma$-field equation (3.7c) is the same as the $N=0$ case, so that all $\zeta$-dependent terms are absorbed into the rescaling (2.9). Second, all $\partial \zeta$-terms arising after the rescaling (2.9) in (3.7a) and (3.7b) contain the factor $\partial \phi$, which is negligibly small for constant or slowly varying $\phi$-field.

Let us consider the vacuum as a de Sitter space represented as a spatially flat FRW space: $\mathrm{d} s^{2}=+\mathrm{d} t^{2}-a^{2}\left(\mathrm{~d} x^{2}+\right.$ $\left.\mathrm{d} y^{2}+\mathrm{d} z^{2}\right)$. Then $G=24 H^{4}$ and the gravitational equation

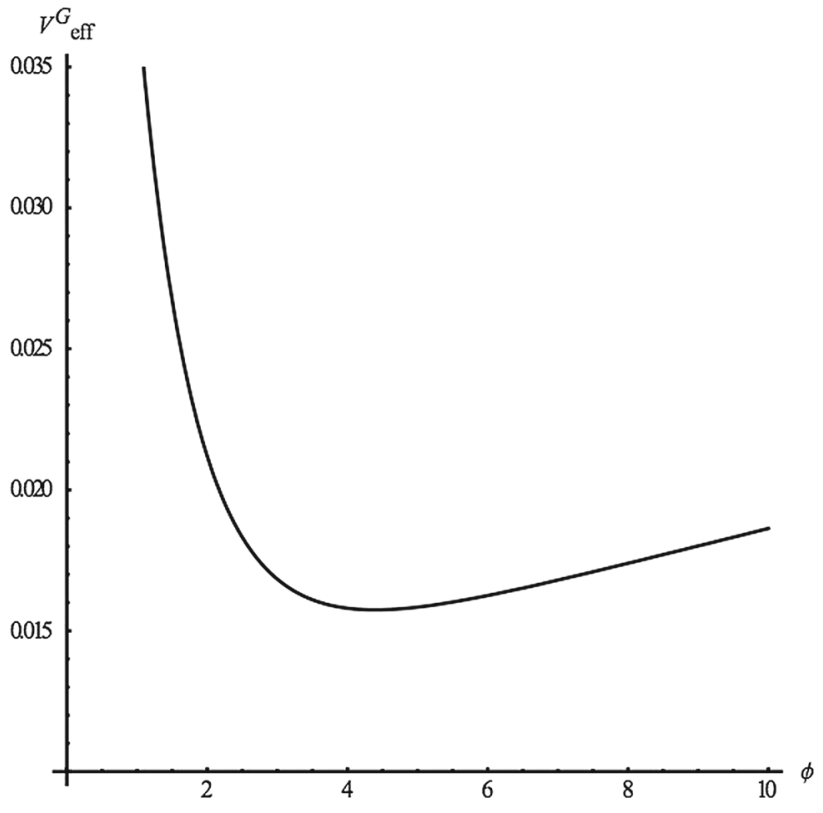

Fig. 2 Graph of $V_{\text {eff }}^{G}$ modified with the GB-term for $M>0$. This graph also has a minimum

determine that $H^{2}=$ (constant) $V_{\text {eff }} .{ }^{5}$ In the presence of the GB-term, the Hubble constant is determined only by $V_{\text {eff }}$. The GB-term effect only enters into the $\phi$-field equation as

$$
V_{\mathrm{eff}}^{\prime}(\langle\phi\rangle)-A V_{\mathrm{eff}}^{2}(\langle\phi\rangle) \doteq 0 .
$$

where $A$ is a constant. Using the form for $V_{\text {eff }}(\langle\phi\rangle)$ in (2.12), we find that the above equation can be written as the extremum of a new effective potential

$$
\begin{aligned}
V_{\mathrm{eff}}^{\mathrm{G}}(\langle\phi\rangle)= & +\frac{A\langle\phi\rangle}{16 M f_{2}}-\frac{A}{64 M \alpha f_{2}}\left(f_{1}+M e^{-\alpha\langle\phi\rangle}\right)^{4} \\
& +\left(\frac{1}{4 f_{2}}-\frac{A f_{2}^{2}}{32 M \alpha f_{2}}\right)\left(f_{1}+M e^{-\alpha\langle\phi\rangle}\right)^{2} \\
& -\frac{A}{16 M \alpha f_{2}}\left[f_{1}^{3}\left(f_{1}+M e^{-\alpha\langle\phi\rangle}\right)+\frac{f_{1}}{3}\left(f_{1}+M e^{-\alpha\langle\phi\rangle}\right)^{3}\right] .
\end{aligned}
$$

We see that the new potential stabilizes the effective potential for positive $M$, which for zero $N$ does not have an extremum; see Fig. 2.

The non-zero cosmological-constant vacuum in the presence of the dilaton-GB coupling does not have the vacuummanifold degeneracy problem in the multi-field situation.

In the case the dilaton couples linearly to the GB-density, the GB-dilaton coupling eliminates flat directions and stabilizes the dilaton. Thus there will be no vacuum-manifold problem in the non-zero cosmological-constant case.

\footnotetext{
5 The exact equation is $G=24\left(H^{2}+k / a^{2}\right)\left(\dot{H}+H^{2}\right)$, which becomes $G=24 H^{4}$ for $k=0$ and $\dot{H}=0$.
} 


\section{Discussion and conclusions}

The enrichment of the TMT by the addition of a linear coupling of the dilaton to the GB-topological density has the effect of stabilizing the dilaton for small cosmological constant at a certain well-defined value of the dilaton field.

We have studied in particular the solution of an effective potential of the form $V_{\text {eff }}=\left(f_{1}+M e^{-\alpha \phi}\right)^{2} /\left(4 f_{2}\right)$, where $f_{1} / M>0$, before the GB-dilaton coupling is introduced. After the GB-dilaton coupling is introduced, this same $V_{\text {eff }}$ determines the value of the cosmological constant for a constant $\phi$, but the equilibrium value of $\phi$ is determined by another potential $V_{\mathrm{eff}}^{G}$, which does have an extremum (which is a minimum).

A related question concerns the lifting of the 'vacuummanifold degeneracy' in the TMT in the multi-field case, when, e.g., we have in addition to the dilaton a Higgs field, etc. In this case given that $V_{\text {eff }}$ is a perfect square, the vacuum for the Higgs and dilaton, even for the case there is dilaton-GB coupling is found to be defined by $V_{\text {eff }}=0$, which defines a manifold in the multi-field case. In the nonzero cosmological-constant case, this degeneracy is absent, furthermore, in the presence of the GB-dilaton couplings, as we have seen, we obtain further stabilization of the dilaton in the single-field case. In the multi-field case with dilatons and Higgs fields, we expect full stabilization and unique vacuum after spontaneous breaking of gauge symmetries. Some aspects of the theory could be further analyzed, for example the effects of matter on the coupling of the dilaton. In [38] for example we have found that, for a high matter density, the dilaton decouples from matter, one could study if such properties remain when the dilaton-GB coupling is added. In Ref. [7], we also obtained another way to give the dilaton a mass generated from instantons, which is another way of stabilizing the vacuum independent of this one, which could also solve the vacuum-manifold degeneracy problem, although this one breaks discrete symmetries like $\mathrm{P}$ and $\mathrm{CP}$ symmetries, since it relies on the coupling of the dilaton to a gauge field topological density that changes sign under parity, but since the dilaton is a scalar, such a term breaks the parity symmetry. It would be interesting to study the full effects of the couplings of the dilaton to both topological densities.

We have so far shown that the cosmological constant is controlled to a desirable amount based on TMT. The reader may wonder about the quantum corrections to the classicallevel cosmological constant. Even though we do not complete our answer to this question, we mention the important points not to be overlooked.

First, $n o$ theory except for superstring theory [44], has ever formulated consistent quantum corrections in gravitational theories. Except for superstring with zero cosmological constant [44], quantum gravity theory is not renormalizable, so that there are no commonly accepted prescriptions for quan- tum corrections. Therefore, at the present time, the problem of quantum corrections is a common problem to any gravity theory dealing with a non-zero cosmological constant.

Second, the quantum corrections may well be controlled by scale invariance. In fact, two of the authors (HN and SR) have proposed a scale-invariant theory [45-50], in which the underlying scale invariance excludes considerable number of counter-terms [51]. In other words, compared with the conventional general relativity, scale-invariant theory [45-51] drastically reduces possible counter-terms. From this viewpoint, it is not far-fetched to expect that the quantum behavior of scale-invariant theory [45-51] is much more controllable than general relativity. Therefore, combining TMT [5] with scale-invariant theory [45-51], the quantum corrections to the cosmological constant are expected to be more suppressed than conventional gravity theories.

Third, as we have mentioned, the Two Measures Theories enjoy the infinite-dimensional group of shifts of the measure fields $\varphi_{a}: \varphi_{a} \rightarrow \varphi_{a}+f_{a}\left(L_{1}\right)$, where $f_{a}\left(L_{1}\right)$ are arbitrary differentiable functions of the Lagrangian $L_{1}$, which is the Lagrangian that couples to the metric-independent measure. This symmetry is valid only if the metric-independent measure remains linear in the effective Lagrangian. So if the quantum corrections were to respect this infinite-dimensional symmetry, the structure of the two measures theories will be preserved even after quantum corrections, and also the general consequences will remain, like the fact that the effective potential is a perfect square will remain and therefore the natural zero vacuum energy density will still be obtained. Intrinsically related to the structure of the TMT is an additional symmetry, not of the full theory, but of the vacuum with zero cosmological constant [52,53]. Symmetries of this type for the zero cosmological-constant state have been searched by many authors, but in the two measures theories they arise without constructing the theory specially for this purpose; for further references see [52,53].

Preserving the structure of TMT does not impose restrictions on counter-terms, as long as they are introduced in a way consistent with this structure in the original frame. When going to the Einstein frame we obtain a reorganization of the interactions, for example a cosmological constant in the original frame enters in the Einstein frame in the denominator of the effective potential of the scalar fields. So a big cosmological constant in the original frame is not necessarily an undesirable feature; we in fact use such a possibility when discussing the cosmological see-saw mechanism in TMT.

Fourth, one may observe that although the models we have discussed have scale invariance, scale symmetry breaking is obtained through the integration of the measure-field equations of motion and the effects considered in this paper survive this scale symmetry breaking. Further scale symmetry breaking from quantum corrections, if they preserve the 
structure of the TMT, are also expected to preserve the main results of this paper.

Finally, we also would like to recall that the theorem by Weinberg $[1,2]$ stating that it is impossible to solve the CCP without invoking fine-tuning does not apply in the TMT [5, 54], because here the assumptions that Weinberg used do not apply.

Acknowledgements One of the authors (EG) gratefully acknowledges the hospitality at CSULB where this work was carried out.

Open Access This article is distributed under the terms of the Creative Commons Attribution 4.0 International License (http://creativecomm ons.org/licenses/by/4.0/), which permits unrestricted use, distribution, and reproduction in any medium, provided you give appropriate credit to the original author(s) and the source, provide a link to the Creative Commons license, and indicate if changes were made.

Funded by $\mathrm{SCOAP}^{3}$.

\section{References}

1. S. Weinberg, The cosmological constant problem. Rev. Mod. Phys. 61, 1 (1989)

2. Y.J. Ng, The cosmological constant problem. Int. J. Mod. Phys. D 1, 145 (1992)

3. B.K. Gibson, T.S. Axelrod, M.E. Putman, N. Bahcall, J.P. Ostriker, S.J. Perlmutter, P.J. Steinhardt, For reviews of this subject, see for example, M.S. Turner in the third Stromlo Symposium: The Galactic Halo, ASP Conference Series, vol. 666, 1999, (eds). Science 284, 1481 (1999)

4. S. Weinberg, The cosmological constant problems. Talk given at Conference: C00-02-23, pp. 18-26 Proceedings. arXiv:astro-ph/0005265

5. E.I. Guendelman, A.B. Kaganovich, Phys. Rev. D 60, 065004 (1999). arXiv:gr-qc/9905029

6. E.I. Guendelman, Scale invariance, new inflation and decaying lambda terms. Mod. Phys. Lett. A 14, 1043 (1999). arXiv:gr-qc/9901017

7. E.I. Guendelman, H. Nishino, S. Rajpoot, Phys. Lett. B 732, 156 (2014). arXiv:1403.4199 [hep-th]

8. E. Guendelman, H. Nishino, S. Rajpoot, Two-measure theory with 3rd-rank antisymmetric tensor for local scale symmetry breaking. (To appear in Phys. Rev. D)

9. E. Guendelman, H. Nishino, S. Rajpoot, Phys. Rev. D 87, 027702 (2013)

10. E.I. Guendelman, Class. Quant. Gravit. 17, 3673 (2000)

11. E.I. Guendelman, Phys. Rev. D 63, 046006 (2001)

12. E.I. Guendelman, A.B. Kaganovich, E. Nissimov, S. Pacheva, Phys. Rev. D 66, 046003 (2002). arXiv:hep-th/0203024

13. E.I. Guendelman, A. Kaganovich, E. Nissimov, S. Pacheva, Phys. Rev. D 72, 086011 (2005). arXiv:hep-th/0507193

14. H. Nishino, S. Rajpoot, Phys. Lett. B 736, 350 (2014)

15. E.I. Guendelman, Phys. Lett. B 580, 87 (2004). arXiv:gr-qc/0303048

16. E. Guendelman, E. Nissimov, S. Pacheva, M. Vasihoun, Dynamical volume element in scale-invariant and supergravity theories. Bulg. J. Phys. 40, 121 (2013). arXiv:1310.2772 [hep-th]

17. E.I. Guendelman, A.B. Kaganovich, Neutrino generated dynamical dark energy with no dark energy field. Phys. Rev. D 87, 044021 (2013). arXiv:1208.2132 [gr-qc]

18. H. Nishino, S. Rajpoot, Mod. Phys. Lett. A 21, 127 (2006)
19. E. Lim, I. Sawicki, A. Vikman, Dust of dark energy. JCAP $\mathbf{1 0 0 5}$ 012 (2010)

20. S. Capozziello, J. Matsumoto, S. Nojiri, S.D. Odintsov, Phys. Lett. B 693, 198 (2010). arXiv:1004.369 [hep-th]

21. E.I. Guendelman, D. Singleton, N. Yongram, A two measure model of dark energy and dark matter. JCAP 1211, 044 (2012). doi:10. 1088/1475-7516/2012/11/044. arXiv:1205.1056 [gr-qc]

22. Stefano Ansoldi (ICRA, Pescara and INFN, Udine and Udine U.), Eduardo I. Guendelman. Sep 2012. Unified dark energy-dark matter model with inverse quintessence. JCAP 1305, 036 (2013). doi:10.1088/1475-7516/2013/05/036. arXiv:1209.4758 [gr-qc]

23. E. Guendelman, E. Nissimov, S. Pacheva, Unified dark energy and dust dark matter dual to quadratic purely kinetic k-essence. Eur. Phys. J. C 76, 90 (2016). arXiv:1511.07071 [gr-qc]

24. E. Guendelman, E. Nissimov, S. Pacheva, Dark energy and dark matter from hidden symmetry of gravity model with a nonriemannian volume form. Eur. Phys. J. C 75, 10-472 (2015). doi:10. 1140/epjc/s10052-015-3699-8. arXiv:1508.02008 [gr-qc]

25. S. del Campo, E.I. Guendelman, A.B. Kaganovich, R. Herrera, P. Labrana, Phys. Lett. B 699, 211 (2011). arXiv:1105.0651 [astroph.CO]

26. S. del Campo, E.I. Guendelman, R. Herrera, P. Labrana, Emerging universe from scale invariance. JCAP 1006, 026 (2010). arXiv: 1006.5734 [astro-ph.CO]

27. E. Guendelman, R. Herrera, P. Labrana, E. Nissimov, S. Pacheva, Gen. Relat. Gravit. 47, 10 (2015). doi:10.1007/ s10714-015-1852-1. arXiv:1408.5344 [gr-qc]

28. E.I. Guendelman, Int. J. Mod. Phys. A 26, 2951 (2011). arXiv:1103.1427 [gr-qc]

29. E.I. Guendelman, Gravitational theory with a dynamical time. Int. J. Mod. Phys. A 25, 4081-4099 (2010). doi:10.1142/ S0217751X10050317. arXiv:0911.0178

30. David Benisty, E.I. Guendelman, Radiation like scalar field and gauge fields in cosmology for a theory with dynamical time. Mod. Phys. Lett. A 31, 33 (2016). doi:10.1142/ S0217732316501881. arXiv:1609.03189 [gr-qc]

31. D. Benisty, E.I. Guendelman, Interactive diffusive unified dark energy and dark matter from scalar fields. arXiv:1701.08667 [grqc]

32. A. Chamseddine, V. Mukhanov, JHEP 1311, 135 (2013). arXiv: 1308.5410

33. A. Chamseddine, V. Mukhanov, A. Vikman, JCAP 1406, 017 (2014). arXiv:1403.3961

34. M. Chaichian, J. Kluson, M. Oksanen, A. Tureanu, JHEP 1412, 102 (2014). arXiv: 1404.4008

35. A.O. Barvinsky, Dark matter as a ghost free conformal extension of Einstein theory. JCAP 1401, 014 (2014). doi:10.1088/1475-7516/ 2014/01/014. arXiv:1311.3111 [hep-th]

36. R. Myrzakulov, L. Sebastiani, S. Vagnozzi, Eur. Phys. J. C 75, 444 (2015). arXiv: 1504.07984

37. S. Tsujikawa, M. Sami, See, e.g., JCAP 0701, 006 (2007). doi:10. 1088/1475-7516/2007/01/006. arXiv:hep-th/0608178

38. E.I. Guendelman, A.B. Kaganovich, Ann. Phys. 323, 866 (2008). arXiv:0704.1998 [gr-qc]

39. H.J. Schmit, D. Singleton, J. Math. Phys. 54, 062502 (2013). arXiv: 1212.1769 [gr-qc]

40. H. Niu, D.G. Pak, Non-topological Gauss-Bonnet type model of gravity with torsion. arXiv:0709.2109 [gr-qc]

41. G.W. Gibbons, S.W. Hawking, Phys. Lett. B 76, 430 (1978)

42. G.W. Gibbons, S.W. Hawking, Commun. Math. Phys. 66, 291 (1979)

43. E.I. Guendelman, O. Katz, Class. Quant. Gravit. 20, 1715 (2003). arXiv:gr-qc/0211095

44. J.H. Schwarz, M. Green, See, e.g., 'Superstring Theory', vols. I and II (Cambridge University Press, Cambridge, 1986) 
45. H. Nishino, S. Rajpoot, Broken scale invariance in the standard model. Phys. Rev. D 79, 125025 (2009). arXiv:hep-th/0403039

46. H. Nishino, S. Rajpoot, Broken scale invariance in the standard model. AIP Conf. Proc. 881, 82 (2007). (Melville, New York, 2006)

47. H. Nishino, S. Rajpoot, Weyl's scale invariance: inflation, dark matter and dark energy connections. in Proceedings of Dark Side of Universe, eds. Shgabaan Khalil, Egypt (2008)

48. H. Nishino, S. Rajpoot, Comment on shadow and non-shadow extensions of the standard model. arXiv:hep-th/0702080

49. H. Nishino, S. Rajpoot, Comment on electroweak higgs as a pseudo-goldstone Boson of broken scale invariance. arXiv:0704.1836 [hep-ph]

50. H. Nishino, S. Rajpoot, Comment on papers by Foot, Kobakhidze, McDonald and Volkas relating to scale invariance symmetry. arXiv:0712.2487 [hep-ph]
51. H. Nishino, S. Rajpoot, Class. Quant. Gravit. 28, 145014 (2011)

52. E.I. Guendelman, A.B. Kaganovich, Transition to zero cosmological constant and phantom dark energy as solutions involving change of orientation of space-time manifold. Class. Quant. Gravit. 25, 235015 (2008). doi:10.1088/0264-9381/ 25/23/235015. arXiv:0804.1278 [gr-qc]

53. E.I. Guendelman, A.B. Kaganovich, Symmetry supporting a transition to zero cosmological constant state. arXiv:0707.4286 [hep-th]

54. E.I. Guendelman, A.B. Kaganovich, Fine tuning free paradigm of two measures theory: k-essence, absence of initial singularity of the curvature and inflation with graceful exit to zero cosmological constant state. Phys. Rev. D 75, 083505 (2007). doi:10.1103/ PhysRevD.75.083505. arXiv:gr-qc/0607111 